\title{
ORQUESTA DE RECICLADOS DE CATEURA: MÚSICA QUE ENSEÑA MÁS ALLÁ DEL CONTEXTO DE LA BASURA
}

Luana Wunsch(*)

Entrevista realizada con el Profesor Favio Hernán Chávez Morán, fundador e idealizador de la Orquesta de Reciclados de Cateura.

Favio Hernán Chávez Morán - Argentino, que vive desde niño en Paraguay. Ingeniero en Ecología Humana, Universidad Nacional de Asunción. Filósofo, Universidad Católica de Asunción. En la música estuvo en el Instituto Municipal de Arte de Asunción y de Dirección Orquestal con Miguel Ángel Gilardi, León Burke y Luis Szarán. Desde 2001 desarrolla proyectos que trabajan con residuos domiciliarios en general, lo que incluye el Proyecto Procicla, 2006, para el Banco Interamericano de Desarrollo, dentro del Vertedero Cateura de Asunción, como técnico y responsable de educación formal, interactuando con las diversas asociaciones de gancheros (recicladores) de dicho lugar. En el año 2008, crea la Orquesta de Instrumentos Reciclados de la cual se constituye Director hasta hoy. Fue Coordinador Académico del Proyecto Sonidos de la Tierra y fundador de la Orquesta de Cámara de Carapeguá, de la Orquesta de Cámara de la Universidad Católica de Carapeguá de las que sigue siendo su Director, y la Orquesta del $9^{\circ}$ Dpto. de Paraguarí. Reconocido expositor en conferencias como TEDx, obtiene visibilidad mundial como uno de los proyectos con mayor alcance inclusivo y contextualizado.

\section{INTRODUCCIÓN}

Para hablar de uno de los proyectos de inclusión social en el área de la música y de la educación en América del Sur a los que más se hace referencia, es necesario que nos ubiquemos en el segmento social que este alcanza. Cateura es el nombre del mayor vertedero de Asunción, Paraguay, que desde la década de 1980 enfrenta graves problemas como falta de saneamiento básico y especialmente, inundaciones y sus consecuencias debido a la precaria infraestructura de las viviendas.

\footnotetext{
${ }^{(*)}$ Doutora em Educação (Universidade de Lisboa, validação brasileira pela Universidade Federal de Pelotas-RS, 2013). Professora do Programa de Pós-Graduação Stricto Sensu em Educação Mestrado Profissional: Educação e Novas Tecnologias (PPGENT) da UNINTER. E-mail: 1priscila@gmail.com.
} 
Cuando se conversa con los moradores de la región, se puede constatar un cambio expresivo cuando surge el tema "los últimos quince años viviendo en Cateura". Entre los canteros, pequeños comerciantes, empleadas domésticas y cocineros, muchos de los que antes apenas sobrevivían del trabajo con / en la basura dan testimonios de mejoría, no solo en lo que se refiere a la calidad financiera de vida, sino algo que definen con una palabra, que aparece a menudo: la esperanza.

Para nosotros, investigadores y profesionales de la educación, este hecho es intrigante. Al final, el abandono ocasionado por las crisis políticas y económicas que asolan a América Latina, afecta directamente a nuestros niños y jóvenes.

Pero ¿qué ha hecho de manera tan expresiva brotar un sentimiento reverso en una comunidad que nada tiene de diferente de tantas otras en nuestro continente?

Según Favio Chávez, actividades humanizadas. Y fue con esta concepción, que a principios del año 2000, después de una larga jornada de trabajo con la música y con técnicas sobre el medio ambiente, este profesional se torna profesor y realiza un proyecto que desde entonces ha ganado expresividad mundial, que recibió el nombre de "Orquesta de Reciclados de Cateura".

Con el tema "el mundo nos envía basura, nosotros le devolvemos música", Favio decidió compartir su gusto por la música con los niños cuyas familias vivían en el mayor basural o vertedero de Paraguay. Para superar la falta de instrumentos y con la ayuda de muchas personas de la población, empezó a realizar pruebas para crear instrumentos con los recursos disponibles en el propio lugar. Utilizando residuos encontrados en Cateura, los instrumentos empezaron a tomar forma y se convirtieron en instrumentos musicales finamente sintonizados, todos hechos con latas de pintura, tenedores, restos de muebles. Así nació la Orquesta de Instrumentos Reciclados, y los niños del lugar comenzaron a aprender y a tocar Bach, Mozart y Beethoven, además con una afinación acorde al nivel de estos compositores.

Si un proyecto hace tanta diferencia en la sociedad en la que está insertado, es de suprema importancia para la investigación educativa comprenderlo de forma más detallada. Por este motivo, esta investigadora realizó in loco, durante el mes de agosto de 2017, un análisis de su estructura y posibles impactos sociales que de él provienen. En este artículo se presenta, ipsis litteris, una entrevista cualitativa realizada con el ya citado idealizador del proyecto. 


\section{1. ¿Qué es, finalmente, la Orquesta de Reciclados de Cateura?}

Si usted le pregunta a cualquier persona de Asunción, ella le dirá que es un proyecto que hace instrumentos musicales con basura reciclada. Yo digo que es más, es una estructura que abarca conceptos de música, de educación y de vivienda.

Comenzamos en 2012 con once niños de una comunidad que estaba por debajo del nivel de pobreza, en el medio del mayor vertedero de basura de Asunción, la capital, recibiendo casi 500 toneladas de desechos por día. Con el apoyo de algunos familiares buscábamos encontrar, en ese contexto, recursos que pudieran dar la motivación necesaria a aquellos niños para ser los protagonistas de algo. Para mí, nada es más íntimo y fascinante que la música. Fue así como la basura se convirtió en violines, violas, chelos (violonchelos), contrabajos, guitarras, flautas, saxofones, trompetas e instrumentos de percusión. De esta forma empieza la base musical del proyecto.

Con el pasar de los años estos niños han crecido, tocan instrumentos reciclados y también instrumentos tradicionales. Muchos de ellos actúan como profesores de la Orquesta, que actualmente cuenta con 300 niños. Constatamos durante todo este tiempo, que la música que nuestros niños producían era única, que superaron la historia de los compositores, que cada instrumento, cada tema hacía que estos músicos fueran protagonistas. Que comprendieron su papel durante su aprendizaje. Una orquesta está compuesta por varios sonidos, pero cada uno tiene la responsabilidad de hacer que su sonido esté bien y que forme parte de este rompecabezas. Este movimiento individual y colectivo, se encaja, para mí, en la base educativa.

Pero ¿cómo podemos tener buenos ciudadanos, buenos alumnos, buenos instrumentistas, si sus casas no tienen saneamiento básico o si todo lo habían perdido en las inundaciones? Poco a poco, con ayuda de donantes, en su mayoría personas físicas, logramos a través del proyecto construir o reformar la vivienda, su hogar, de muchos de los alumnos que están con nosotros desde el principio. Entra, por lo tanto, la cuestión de la vivienda.

2. ¿En 2008, cuando todo empezó, usted esperaba sacar a los niños de la pobreza y convertirlos en músicos profesionales e internacionales? ¿Después de estos años, cree haberlo conseguido?

No, jamás. La intención del proyecto nunca fue formar músicos profesionales, sino ciudadanos que tuvieran opción de elección en su vida. Formar personas que respondieran a sus necesidades a partir de lo único que tenían a su alrededor, la basura. Ayudar a concientizar a las 
personas que tenían, en su mayoría, las casas hechas y amuebladas solamente con lo que encontraban en la basura, hacer arte con personas que la sociedad marginaliza, hacer arte con cosas que la sociedad descarta.

La música es un fuerte punto de apoyo en la formación humana. Pero en Cateura, una guitarra puede costar más caro que una casa. ¿Entonces qué hacer para que esto fuera posible?

La inclusión social en la orquesta fue más allá de los niños, los familiares, muchos de ellos, ayudaron en la construcción. Observamos que utilizar las habilidades que las personas tienen, es ser conscientes de que el aprendizaje puede surgir de todas partes, y esto debe ser significativo para la realidad que conocen y en donde estarán. Por lo tanto, los propios padres y abuelos que construyeron sus casas con materiales que encontraron en la basura, con estos mismos materiales ayudaron a construir los instrumentos que tanto necesitábamos.

Lo que hizo que se creara una asociación de padres, la transformación que veo es sobre todo, familiar.

\section{¿Internacional?}

Todos los que están con nosotros desde el principio, acabaron transformándose. Tocamos, por invitación, en más de 40 países en todos los continentes, con orquestas de renombre, con bandas conocidas. Pero muchos de ellos no quieren seguir la carrera de músicos profesionales, quieren ser abogados, administradores, informáticos. Y lo serán. Es lo que se logra cuando se aprende que la educación es la herramienta que nos da el poder de elección en el futuro.

Yo quería tener el poder de sacar a todos de la pobreza, pero no lo tenía, no lo tengo. Yo deseaba, y aún deseo, incluir ciudadanos en una sociedad más justa. Apoyarlos para que deseen mejorar el medio en el que viven y en el que pronto actuarán. Para mí la música era un factor de confort, de inspiración, quería que ellos también tuvieran este punto de apoyo.

Como en aquella realidad era imposible comprar instrumentos a los que ni yo, ni muchas personas tenían acceso. Tuvimos que repensar el concepto de lo que era instrumento musical.

Lo que siempre he tenido claro es que todos merecemos lo mejor. Entonces, si íbamos a hacer música con basura, yo quería los mejores instrumentos hechos de basura. No eran solo aquellos que vemos en escuelas para diversión, era y es, todo en serio. Quería enseñar notas musicales suaves e intensas. 


\section{3. ¿Qué cree que lo hace ser uno de los profesores más citados acerca de la inclusión social en América del Sur?}

No trabajamos con educación formal, pero quiero que mis alumnos lean partituras, de la misma como una escuela privada enseñaría. Un contenido de calidad es importante para una buena música, es importante para todo, es importante para la vida.

Les quitamos, desde siempre, el concepto de lástima que ellos podrían acarrear debido a sus vivencias e intentamos, con estas mismas experiencias, siempre transformarlos en alguien capaz para vivir con un elemento motivador y de promoción para los niños y jóvenes, de modo que estos vivan experiencias que los ayuden a aprender, a permanecer en la escuela, a desarrollar su creatividad y a tener acceso a oportunidades para su futuro.

No soy un profesor que actúa solo, cuento con la ayuda de las familias, nadie aprende solo. Y sin familia el aprendizaje es más doloroso, en todas las realidades.

Creo que es porque la orquesta de Cateura avanza más allá de la música y entra en el campo de las prácticas educativas. Para mí, estas prácticas no pueden, incluso en la educación en escuelas regulares, estar solo dentro de las cuatro paredes del aula. Es necesario tener en cuenta la experiencia de los alumnos y la experiencia de los padres de estos alumnos, para que el contexto de la vida de quien aprende sea el inicio del proceso de aprendizaje, el medio y el objetivo a ser alcanzado, no solo asignaturas aisladas, con contenidos sin función.

Creo que esto es fundamental para la educación social en Cateura, en el Paraguay, en América del Sur, en los Estados Unidos y en Finlandia.

\section{4. ¿Entonces un proceso de aprendizaje significativo es la propuesta pedagógica de la} Orquesta de Reciclados de Cateura?

Claro, pero la que tiene la cooperación como propuesta. No estamos afiliados a ninguna mantenedora formal de educación y tampoco de la música, por eso nuestra propuesta es hecha por nosotros, para nosotros. No tenemos cargos específicos que piensen en documentos educativos, tenemos una comunidad pensando en la mejoría de la calidad de vida de sus agentes. Este es el punto central de la significación. No medimos en números el impacto del trabajo de la orquesta en el aprendizaje de los alumnos lo medimos por medio de sus actitudes, cuando vemos que la música efectivamente los ayudó a ser más autónomos, conocedores de sí, de los problemas sociales de su región y del mundo, pero también conscientes de que ellos son seres activos en la superación y resiliencia futura. 
Enseñar aquí es mediar, concientizar y mostrar opciones. Creo que esto es un aprendizaje significativo.

5. Una de las palabras más citadas en investigaciones de educación a nivel mundial es la calidad del escenario que se investiga. En el área de la música, así como en la educación, se sabe que para saber lo que realmente significa calidad en el trabajo realizado es necesario analizar muchas variables. ¿Cómo analiza la calidad de los resultados que obtiene con sus músicos? ¿Serían las mismas de las grandes orquestas con instrumentos formales?

Fue todo un proceso para entender que no dejamos nada a desear. No podemos comparar el trabajo realizado aquí con los que se realizan en otros lugares, y eso no está relacionado con nuestros instrumentos hechos de basura.

Explico: Cuando empezamos teníamos once niños, violines y violas elaborados con la basura que estaba presente en sus vidas, porque era la opción alcanzable, posible. Era por una creencia de superación de los aspectos sociales y económicos. Con el pasar del tiempo, constatamos que estos niños no solo superaron dificultades económicas, sino que se volvieron grandes conocedores de música formal, leían e interpretaban partituras a su manera. El sueño de todo maestro, tener un músico que interprete y coloque emoción en lo que toca. Teníamos varios aquí.

Con el tiempo, ganamos visibilidad, tuvimos buenas donaciones de instrumentos formales. Claro que luchamos por recursos todos los días, pero tenemos conciencia de que somos mayores comparados con lo que éramos cuando empezamos.

Para intentar responder a la pregunta, digo que la calidad de lo que hacemos puede ejemplificarse con la siguiente constatación: hoy llegamos a más de 300 niños y jóvenes, no solo de Cateura, sino de toda Asunción. Los incluimos a todos, todos aquellos que quieren aprender buena música, no excluimos. Y podemos ver nuestro avance, para mí poder avanzar es una variable de calidad, cuando cuento que todos los principiantes empiezan aprendiendo con los instrumentos formales, porque son más intuitivos. Los alumnos con más experiencia, más conocedores, pasan a los instrumentos de basura, estos no son obvios.

Para acompañar a la orquesta que hace las presentaciones por el país y por el mundo con los violines, percusión y demás instrumentos de basura es necesario tener fuerza de voluntad, estar dedicado y entender de música. Los instrumentos de basura son también evaluados por los propios alumnos, y se convirtieron en una meta a ser alcanzada.

Nuestro medidor de calidad es la percepción de nuestros alumnos ante la estructura misma de lo que ofrecemos. Ellos quieren estar aquí y recorrer el camino, con metas, sí, pero con 
esperanza y dedicación para la superación. Y claro, cuando estamos en diferentes países, con diferentes lenguas y vemos que aquellos jóvenes que quieren estar en esta propuesta son aplaudidos de pie por legos y por críticos musicales.

6 - Vamos a salir un poco de la cuestión solo pedagógica y entrar en la cuestión de las políticas públicas, de las que un proyecto como éste no puede permanecer ajeno. Conocemos la complejidad que involucra las relaciones entre política y proyectos sociales. Así, es imposible no preguntar, ¿Cómo es la relación de la Orquesta de Reciclados de Cateura con los gobernantes en su país?

Afirmo, no tenemos vínculo político, no tenemos conexión con la captación de fondos públicos. La mayoría de las veces nuestra relación es amigable. Sería inútil decir que no somos algo con un impacto social, los medios de comunicación siempre muestran las cosas que hacemos, tenemos muchos visitantes de otros países que están en Asunción para conocer nuestra historia de cerca. Somos vistos, como en cualquier lugar del mundo, la política en Paraguay tiene diferentes bases de pensamiento y por lo tanto, algunos gobernantes quieren estar más cerca, otros quieren estar y que estemos lejos.

Como para mantenernos necesitamos donaciones, la mayoría viene del extranjero, de muchos amantes de la música. Queremos ayudar a los nuestros, los de nuestra comunidad, pero no olvidamos que tenemos que pensar en la mejoría de nuestro país. No estamos aislados. No se puede educar de forma alienada y aislada, aunque muchos no entienden eso.

¿Si somos comprendidos? En la mayoría de los encuentros con los gestores, sí. En los demás casos, no desistimos.

Un ejemplo, viajamos mucho y somos muchos, casi nunca menos de veinte, entre los jóvenes músicos y padres de apoyo, somos conscientes de que la logística no es barata. Exceso de equipaje por los instrumentos, los latones de pintura de reciclados son pesados. Una de nuestras premisas es que los pasajes y los alojamientos sean emitidos por agencias paraguayas, siempre. Sea un viaje a una ciudad en los alrededores de Asunción o hacia los Estados Unidos, China, Austria. Somos de aquí y queremos mover la economía de aquí.

Ese es un ejemplo, pero aunque sean pequeñas acciones, lo hacemos. Incluir socialmente no es solo para nosotros, sirve como principio de buena convivencia. A veces lo conseguimos. 
7. Todo es muy intenso. Las conquistas fueron muchas y de gran impacto en la vida de los que están involucrados, de forma positiva. Se ve cuando estamos aquí, conversando con la comunidad, con los alumnos, tomando café con algunos padres. Pero, ¿cuáles fueron los principales obstáculos? ¿Ha tenido algún momento en el que haya creído que debería parar?

Que debería parar, no. Momentos en que estuvimos cansados, muy cansados, muchas veces.

Esto no cambió solamente la vida de las familias de los alumnos, sino mi vida y la de mis familiares. Todo cambió desde el primer violín hecho de latas.

Desafíos y superaciones tenemos cada minuto, actuamos con personas y ellas no son seres conformados, no aquellas con las que estamos.

Puedo contar sobre el llanto cuando enfrentamos la pérdida de muchos instrumentos, muchos materiales, fue un momento doloroso. Con mucho esfuerzo, conseguimos construir este ambiente en el que estamos hoy, con más salas, con un lugar adecuado para guardar los instrumentos de reciclados y los formales.

La región de Cateura, hoy, no es solo un vertedero de basura en el que la gente vivía, ahora es un lugar habitable. Muchos basureros cambiaron de profesión, ganan un poco más, y muchos vinieron a vivir aquí, sin haber trabajado nunca con el mal olor y con los animales que provienen de la suciedad.

Pero esto no significa que la región tenga toda la infraestructura mínima deseada. Todavía enfrentamos muchas cosas en este sentido.

Enfrentamos la cuestión financiera, todo debe estar bien planificado y organizado para que todos tengan acceso a lo que podemos ofrecer. Y esto es bastante desgastante. Pagamos el precio por creer que no queremos estar con mantenedoras políticas, como ya lo he dicho.

Todos ayudan, pero tenemos una comisión de padres y profesores que ayudan en los asuntos más burocráticos, logísticos. Es todo muy difícil, pero no lo supero solo, es por eso hemos llegado hasta a China.

\section{8. ¿Para finalizar, qué viene después del sonido de la basura?}

Vendrá la mente abierta al mundo, a las posibilidades, al poder de elección. Pero no por la basura, que es solo un instrumento, como podría serlo un teléfono móvil de última generación. Es por lo que hacemos con la basura, es por el intercambio de experiencias que realizamos con este 
instrumento. Es por las amistades que conquistas a partir del sonido. Es por nuestra conexión con el ambiente en el que estamos. Es por la conciencia de que así como algunos tienen acceso a los celulares de última generación, nosotros solamente teníamos acceso a la basura, pero no por eso somos menos o más.

Vendrán futuros músicos, futuros maestros, futuros productores musicales; pero vendrán también futuros abogados, futuros médicos, futuros comerciantes, futuros basureros, pero todos con el poder de elección consciente sobre qué camino quieren seguir. Nada de estar marginados, nada de ser periféricos, todos de acuerdo en que la música educa, transforma y amplía los logros, de que hay que saber trabajar en equipo y que sus acciones interfieren en las acciones de los demás. Vendrán nuevos ciclos, nuevas dificultades, nuevas superaciones, pero todo con arte y, por lo tanto, con más belleza y movimiento. 


\section{RESUMEN}

La presente entrevista con el profesor Favio Chávez, fundador e idealizador de la "Orquesta de Reciclados de Cateura", expone los presupuestos de sociedad y educación que provienen de este proyecto. Durante una investigación in loco en la comunidad de Cateura, Asunción, Paraguay, en el segundo semestre de 2017, la autora observó la motivación pública para abordar asuntos globales de pobreza extrema. Pero también fue intensamente relatado, por la misma población, la transformación de este espacio social por medio de la música que se produce en su entorno, con instrumentos elaborados con materiales recogidos en el mismo lugar, en el mayor vertedero de basura de Paraguay. Desde esta óptica, se presenta una entrevista, de tipo cualitativo, acerca de la fundación, de las relaciones, de los espacios y de los tiempos que surgen de una acción que no está vinculada a ninguna mantenedora formal de educación, y que hoy se considera como una de las más importantes propuestas de contextualización del arte y la educación de América del Sur.

Palabras clave: Enseñanza de Música, Inclusión Social, Instrumentos musicales reciclados

\section{ORQUESTRA DE RECICLADOS DE CATEURA: MÚSICA QUE ENSINA PARA ALÉM DO CONTEXTO DO LIXO}

\section{RESUMO}

A presente entrevista com o professor Favio Chavez, fundador e idealizador da "Orquestra de Reciclados de Cateura", expõe os pressupostos de sociedade e educação que provêm deste projeto. Durante uma pesquisa in loco na comunidade de Cateura, Assunção, Paraguai, no segundo semestre de 2017, a autora percebeu a motivação pública para abordar questões globais de pobreza extrema. Porém como também é intensamente relatado, pela mesma população, a transformação deste espaço social por meio da música que é produzida ao lado, com instrumentos confeccionados com materiais recolhidos do mesmo modo, no maior aterro do Paraguai. Sob tal ótica, apresenta-se uma entrevista, de cunho qualitativo, acerca da fundação, das relações, dos espaços e dos tempos que emergem de uma ação que não está vinculada a nenhuma mantenedora formal de educação e que hoje é considerada uma das mais relevantes propostas de contextualização de arte e educação da América do Sul.

Palavras-chave: Ensino de Música, Inclusão Social, Instrumentos musicais reciclados.

\section{THE CATEURA RECYCLED ORCHESTRA: MUSIC THAT TEACHES IN THE CONTEXT OF GARBAGE}

\section{ABSTRACT}

The present interview with Professor Favio Chavez, founder and founder of the Orquestra de Reciclados de Cateura, exposes the presuppositions of society and education that come from this project. During an on-site survey in the community of Cateura, Asunción, Paraguay, in the second half of 2017, the author noticed the public motivation to address global issues of extreme poverty. However, the transformation of this social space through the music that is produced alongside it, with instruments made with materials collected in the same way, into the largest landfill in Paraguay, is also intensely reported by the same population. From this point of view, there is an interview of a qualitative nature about the foundation, relationships, spaces and times that emerge from an action that is not linked to any formal provider of education and which today is considered one of the most relevant proposals of contextualization of art and education of South America.

Keywords: Music Teaching, Social Inclusion, Recycled Musical Instruments 